\title{
THE RESULTS OF TREATMENT IN 1,086 GENERAL PARALYTICS THE MAJORITY OF WHOM WERE FOLLOWED FOR MORE THAN FIVE YEARS
}

\author{
Richard D. Hahn, M.D., ${ }^{1}$ Bruce Webster, M.D. ${ }^{2}$ George Weickhardr, M.D. ${ }^{3}$ \\ Evan Thomas, M.D., ${ }^{4}$ William Timberlake, M.D., ${ }^{5}$ Harry Solomon, M.D., ${ }^{6}$ \\ John II. Stokes, M.D., ${ }^{7}$ Albert IIfyman, M.D., ${ }^{8}$ George Gammon, M.D., ${ }^{9}$ \\ Geraldine A. Gleeson, A.B., ${ }^{10}$ Arthur C. Curtis, M.D., ${ }^{11}$ AND \\ John C. Cutler, M.D. ${ }^{12}$
}

(Received for publication Nov. 26, 1957.)

I AM greatly honored to have been invited to address you as the spokesman for a group of clinics which includes the Bellevue, Boston Psychopathic, St. Elizabeths, Grady Memorial (Emory University), Johns Hopkins, New York, University of Michigan, and University of Pennsylvania Hospitals. The records of 1,086 paretic patients from all these institutions were pooled and analyzed statistically with the assistance of Mrs. Geraldine A. Gleeson and Dr. John C. Cutler of the United States Public Health Service. My chief, Joseph Earle Moore, gave invaluable constructive criticism and advice.

The present study is an evaluation of the therapeutic efficacy of penicillin in 1,086 patients with general paralysis. The possible additive effects of fever therapy are also considered. No untreated control group is available, but the end point in untreated general paralysis, as evidenced by the literature before the introduction of malaria therapy in 1917, is death, usually within a period of 4 years from detection.

To provide for the uniform coding of records at the various cooperating institutions, work sheets of the multiple choice variety were drawn up, and the results transferred to punched cards. The fixed point of reference with respect to time was the so-called "study treatment" date. This was the treatment episode which placed the patient in the study group, whether or not previous treatment had been given elsewhere.

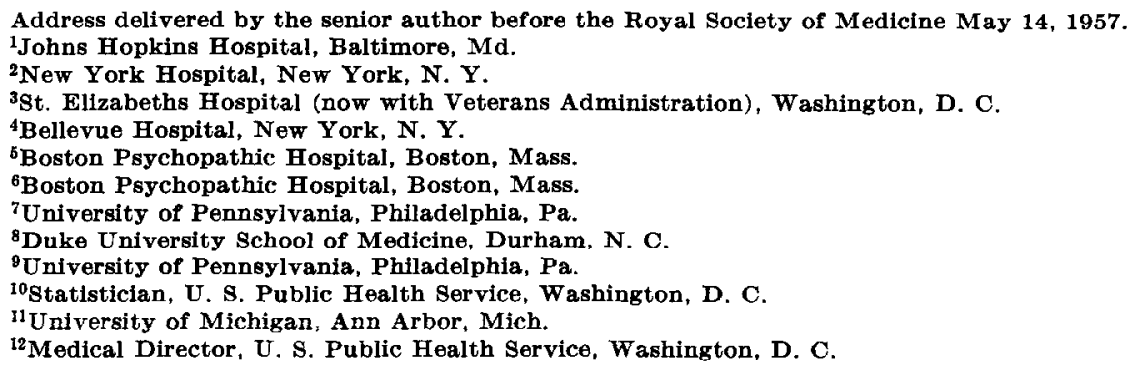


Patients were observed at 3, 6, and 12 months after study treatment, and yearly thereafter. At each observation a worksheet was filled out with a comparison of the status at that time with the status preceding study treatment.

Of the 1,086 patients, 629 were treated with penicillin alone, and 457 with penicillin plus fever therapy, usually induced tertian malaria. The period of observation after treatment is shown in Table $I$. The fact that over 90 per cent of these patients were followed for 1 year or more and 60 per cent for 5 years or more lends a certain validity to the conclusions of the present study.

Table 1. Posttreatment Observation: 1,086 Paretics

\begin{tabular}{ll}
\hline 1 year or more & $92 \%$ \\
3 years or more & $79 \%$ \\
5 years or more & $60 \%$ \\
7 years or more & $34 \%$ \\
\hline
\end{tabular}

Information as to previous treatment was unavailable in 20 per cent of cases Almost 40 per cent of those for whom such information was available had been entirely untreated at any time. Only 5 per cent of patients had received any penicillin. Consideration of the stage of infection when treatment was first given revealed that only 1 out of 10 patients had received any treatment for early syphilis and in no case had therapy included penicillin. A recently published study by the same cooperative group of 765 patients with asymptomatic neurosyphilis followed in most cases more than 5 years after penicillin treatment showed that paresis did not develop under these circumstances within the observed post-treatment period.

The duration of syphilitic infection could not be determined in half of these patients, and in the remainder had a spread, as roughly estimated from the histories provided by psychotic patients, of from 2 to more than 30 years, with the majority falling in the period of 10 to 24 years.

In the present large group of patients, paresis was seen approximately three times as frequently in males as in females, and over twice as frequently in whites as in Negroes. Eighty-five per cent of the patients were in the age group of 30 to 59 years.

The spinal fluid complement fixation test was positive at the time of treatment in all of the 327 previously untreated patients, 85 per cent of whom showed 5 or more cells. In 92 per cent of the same untreated group the blood S.T.S. at the time of treatment was positive.

As shown in Table II, the simple dementing type of psychosis was more frequent than all the others combined.

In Table III is shown the percentage distribution with respect to duration of paretic symptoms at the time of treatment. In paretics the great difficulty of obtaining a reliable history in this regard, even from the patient's family and associates, is well known. A not inconsiderable percentage of the present group failed to give sufficient information for even an estimation of the onset. Ideally, paresis should be promptly diagnosed and as promptly treated. The present 
group represents, by and large, a far different situation with only 16 per cent of the paticnts entering the study with symptoms of 3 months' duration or less. The benefit of treatment reported here may thus be considered to be minimal.

Table II. Type of Psychosis

\begin{tabular}{lr}
\hline & \\
& \\
Simple dementing & $59.8 \%$ \\
Manic, expansive & $18.1 \%$ \\
Depressed & $5.8 \%$ \\
Paranoid & $6.4 \%$ \\
Somatic signs only & $3.2 \%$ \\
& $6.7 \%$ \\
\hline
\end{tabular}

Table III. Duration of Paretic Symptoms

\begin{tabular}{|c|c|}
\hline MONTHS & PER CENT \\
\hline $\begin{array}{c}0-3 \\
4-6 \\
7-12 \\
13-36 \\
37+ \\
\text { Unknown }\end{array}$ & $\begin{array}{l}15.9 \\
10.8 \\
17.9 \\
19.6 \\
15.3 \\
20.5\end{array}$ \\
\hline
\end{tabular}

Table IV. Clinical Severity of Involvement

\begin{tabular}{ll}
\hline \hline & \\
& \\
Severe & $56.3 \%$ \\
Moderate & $21.5 \%$ \\
Mild & $22.2 \%$ \\
\hline
\end{tabular}

In order to provide some basis for prognosis and for comparison of other variables, an attempt was made to estimate the "severity" of clinical paretic involvement at the time of treatment. Arbitrarily the following criteria were considered to be indicative of advanced disease (referred to as group I symptoms): psychotic on simple observation, delusions, disorientation, incontinence, untidy dress, illogical conversation, and inability to perform simple acts of personal toilet. Symptoms considered to indicate less advanced paretic involvement (group II symptoms) included impairment of retention, memory, information, calculation, judgment, insight, speech, handwriting, and the presence of tremors.

Patients who had two or more group I symptoms were classified as having "severe" paresis. Patients who had only one or none of the group I symptoms, but five or more of the group II symptoms, were classified as having "moderate" paretic involvement. Patients who had only one or none of the group I symptoms and less than five of the group II symptoms were classified as having "mild" paretic involvement. The percentage distribution of cases with respect to this classification is shown in Table IV. There are several reasons for believing, 
despite its arbitrary nature, that the classification used is a valid one. It correlates well with the source of cases, as 375 of the 611 cases classified as severe come from the only two psychiatric hospitals among the eight cooperating institutions (St. Elizabeths and the Boston Psychopathic). It also correlates well with the pragmatic factor of ability to work, since only 3 per cent of paretics classified by our criteria as "severe" were able to work at the time of treatment contrasted with 33 per cent of those classified as "moderate" and 53 per cent of those classified as "mild."

Severity of clinical psychosis did not correlate well with estimated duration of paretic symptoms. It is felt that this lack of correlation is more apparent than real and is due at least in part to the unreliability of such historical data concerning duration of symptoms as is obtainable from psychotic patients and/or their frequently unintelligent or unobservant relatives and associates.

I omit details as to the type and amount of penicillin given to these patients except to say that most of them were treated at a time when aqueous penicillin was in vogue. Almost all of them received over 2.4 million units, and a bit over half of them received more than 6 million units. There was a definite tendency to treat clinically "severe" paretics with larger dosage. There was also a similar tendency to add fever therapy to penicillin more frequently in the "severe" paretics. It is of interest that the trend in recent years in the United States has been toward utilication of penicillin alone.

Herxheimer reactions, both clinical and febrile, occurred significantly more frequently after treatment in patients with pleocytosis (of 11 or more cells) than in those without pleocytosis in the spinal fluid. Among the 44 clinical Herxheimer reactions, exacerbation of the psychosis or the development of convulsive seizures were the major problems, occurring in 24 and 6 patients, respectively. One patient died in status epilepticus.

Broadly considered, the results of treatment of paretics may be evaluated from two points of view - the physical and the psychiatric outcome. Utilizing a modified life table technique, all patients were carried through to latest observation, re-treatment, or death. The cumulative probability of death was thus determined. Death was further categorized as to cause, i.e., neurosyphilis or other reasons.

The survivors were then further considered in relation to pretreatment status. In general, two measurements of psychiatric outcome have been utilized. (1) Clinical status of the paretic psychosis after treatment in relation to pretreatment status. In this determination the judgment of the clinician was paramount. The possible categorizations were (a) remission-significantly improved, (b) unchanged, and (c) progression. (2) Work status after treatment in relation to pretreatment work status - a pragmatic yardstick of evaluation largely independent of the vagaries of individual clinical judgment, but subject to a variety of imponderable general extraneous influences such as the business cycle, availability of vocational rehabilitation, and of suitable work, etc. The possible categorizations were (a) able to work, (b) unable to work but not in a mental institution, and (c) in a mental institution. Cases in which inability to work was for reasons other than general paralysis were accounted for. 
In Fig. 1 is shown the cumulative probability of death among 1,086 paretics after treatment with penicillin. The lowermost curve shows the cumulative probability of death from paresis itself. This is only 4 per cent at 1 year, 5 per cent at 2 years, 6 per cent at 4 years, 8 per cent at 6 years, 9 per cent at 8 and 10 years. The second curve, which shows the probability of death from all forms of syphilis, is only slightly higher. The next curve, which shows the probability of death from causes other than syphilis, clearly indicates that even among paretics (after penicillin treatment, of course) the probability of death from syphilis is significantly less than the probability of death from extraneous causes, a startling finding in view of the formerly dread nature of general paralysis.

The top curve shows the probability of death from all causes, syphilis included. This is 8 per cent at 1 year, 12 per cent at 2 years, 17 per cent at 4 years, 23 per cent at 6 years, 28 per cent at 8 years, and 31 per cent at 10 years.

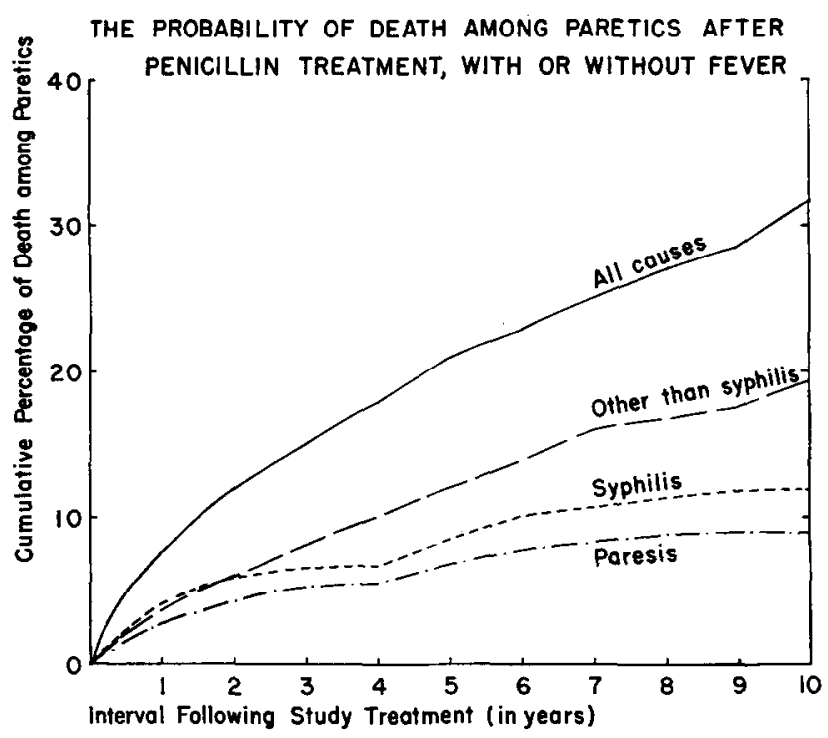

Fig. 1.

Not shown in graphic form are comparable probabilities of death for patients who received only one course of treatment since these probabilities are identical with those shown for the group as a whole. This essential identity of probability of death among those who received one and those who received more than one course of treatment is strong evidence against the necessity for routinely repeated courses of penicillin in the treatment of general paralysis.

As a matter of fact, the total role of penicillin-treated general paralysis as a cause of death is not accurately depicted by any of the curves of Fig. 1, for in addition to deaths due to neurosyphilis and other forms of syphilis, there is an excess mortality due to other diseases among penicillin-treated paretics as compared with the population at large. This excess mortality is doubtless due to 
increased susceptibility as the result of varying degrees of debilitation. Somewhere between the curves depicting the probability of death due to syphilis and the probability of death due to all causes lies the true cost of paresis in terms of death. The difference in death rates between penicillin-treated general paralytics and a similar age, race, and sex group of nonsyphilitics has been estimated by computing specific age, race, sex, and total death rates in our study populationthat is the number of deaths per one thousand person years of observation, and applying them to the 1950 United States population to secure a composite rate adjusted for age, race, and sex. The actual death rate for causes other than syphilis occurring in the United States, in 1950, was compared with the adjusted rate for our study group. The death rate per one thousand per annum was 10.8 for the population as a whole, and 37.7 for the penicillin-treated paretic group. It may thus be concluded that the paretic, even though treated with the best available methods, has nearly four times the death rate of his or her nonsyphilitic age, race, and sex counterpart. The best treatment of paresis remains its prevention by the prompt detection and adequate treatment of early syphilis or asymptomatic neurosyphilis.

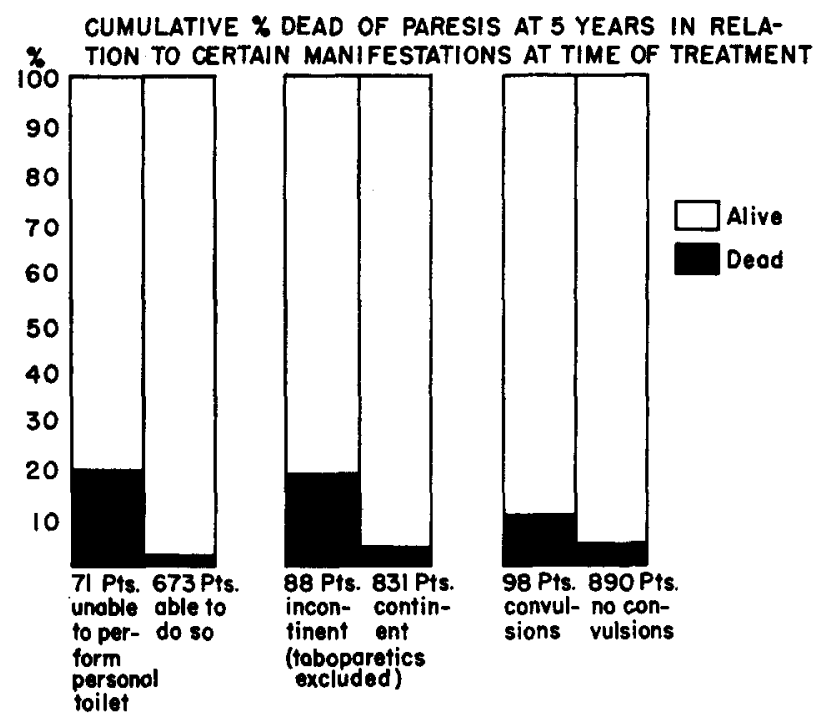

Fig. 2.

As shown in Fig. 2, the presence of certain symptoms at the time of treatment markedly influenced the cumulative per cent of patients dead of paresis at 5 years after treatment. At that time 19 per cent of paretics unable to perform simple acts of personal toilet at time of treatment were dead of paresis as compared with only 2 per cent of patients able to do so. Similarly the presence of incontinence or of convulsions afforded a less favorable prognosis.

As shown in Fig. 3 the cumulative probability of death from paresis at 5 years after treatment was 10 per cent for patients with severe psychosis when 
treated, and zero for patients with moderate or mild psychosis. The cumulative probability of death from causes other than paresis was essentially identical for all three groups.

These data afford further confirmation of the classification of paresis into mild, moderate, and severe, based upon symptoms at the time of treatment. Indeed, it may be stated that the performance of a skilled neuropsychiatric examination at the time of treatment affords the clinician definite prognostic data to a certain extent as to life itself.

In Fig. 4 is shown the clinical status at 7 years after treatment in relation to "severity" of paretic psychosis. In this and subsequent figures, the number of patients followed for the full time interval stated in the chart is shown. Fig. 4 illustrates two important points; first, in mild or early cases better than 80 per cent can be rehabilitated and remain so for at least 7 years; and second, the apparent severity (as we have classified it) of the paretic psychosis at the time of treatment would appear to be of considerable prognostic import.

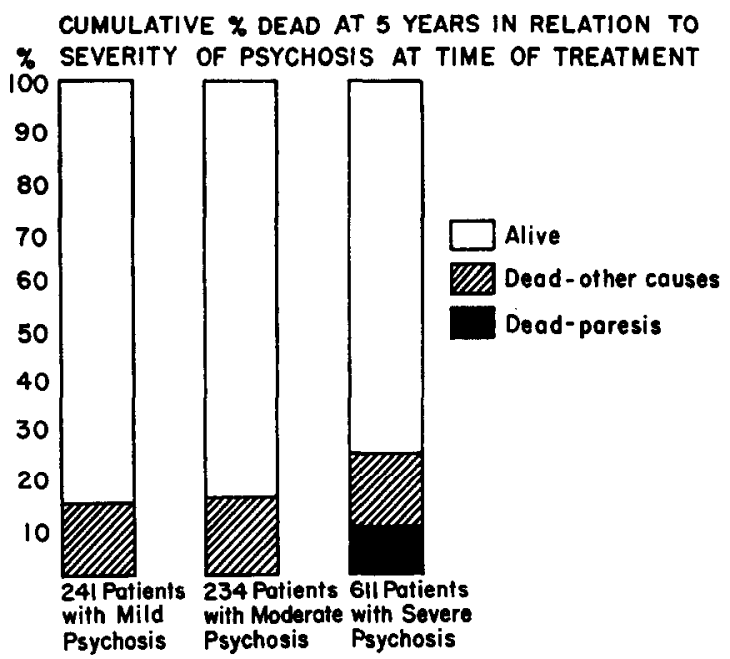

Fig. 3.

In Fig. 5 the per cent of patients in a psychiatric institution 7 years after treatment according to severity of psychosis is shown and compared with pretreatment status. Five times as many patients with apparently severe psychosis at time of treatment were in a psychiatric institution 7 years later as compared with patients originally classified as having moderate or mild psychosis. In all groups results were excellent. Progression of psychosis and death due to general paralysis occurred practically altogether in the severe group.

In Fig. 6 is shown the per cent of patients able to work 6 years after treatment in rclation to work status at time of treatment. It illustrates the considerable prognostic importance of ability to work at the time of treatment but also shows 
that with penicillin therapy one out of three of the least favorable group (institutionalized paretics) can be rehabilitated for some form of work. Of perhaps equal significance is the length of time which has elapsed since the patient has

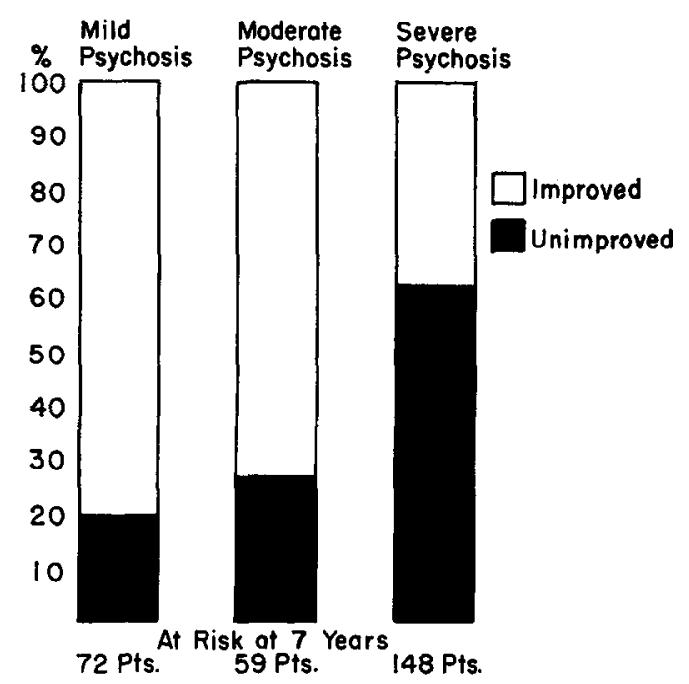

Fig. 4.-Clinical status at 7 years after treatment.
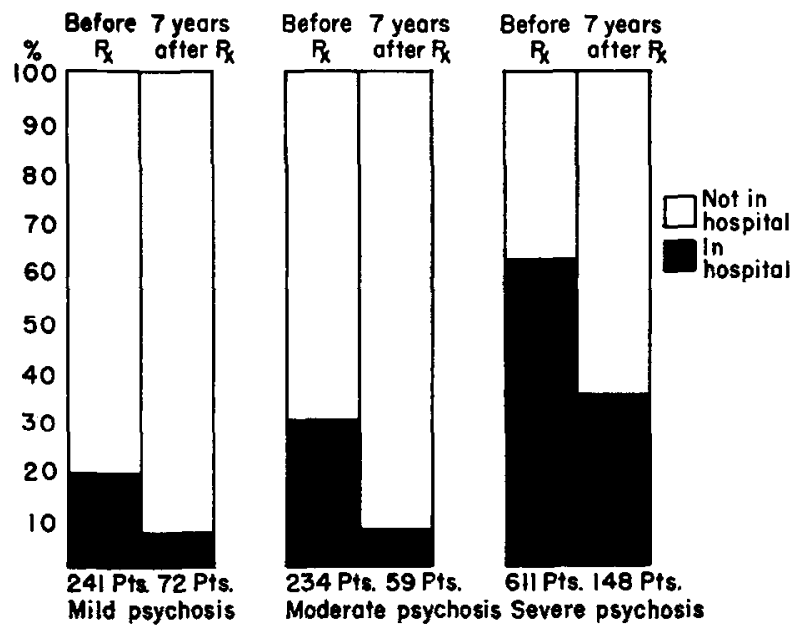

Fig. 5.-Per cent in psychiatric institution 7 years after treatment in relation to per cent institutionalized at time of treatment.

been able to work at his usual occupational level at the time of treatment, because ability to work 4 years after treatment was inversely proportional and psychiatric institutionalization directly proportional to the length of time decreased work capacity had existed at the time of treatment. 
Due to the small number of cases available after subcategorization, study of ability to work after treatment at the same occupation as before treatment was not feasible. However, among 118 skilled workers and 215 unskilled workers institutionalized at time of treatment, ability to work at some socioeconomic level 5 years after treatment was not significantly different. The clinician's evaluation of the severity of the pre-treatment paretic psychosis correlated well with ability to work 5 years after treatment, as only approximately one out of three severe cases were working at any level 5 years after treatment in comparison with over 80 per cent of moderate cases and over 90 per cent of mild cases.

In Fig. 7 is shown the per cent of patients improved 5 years after treatment in relation to pre-treatment spinal fluid cell count. The higher the pre-treatment spinal fluid cell count, the greater is the percentage of clinical improvement 5 years after treatment. Progression rates and death due to neurosyphilis were, however, slightly higher in patients with pre-treatment spinal fluid pleocytosis.

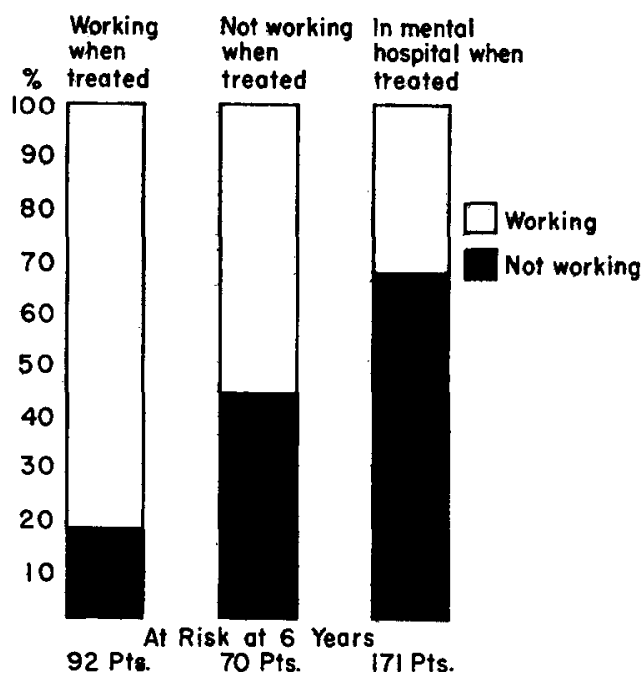

Fig. 6. - Per cent able to work 6 years after treatment.

An elevated pre-treatment spinal fluid cell count indicates an active inflammatory process more susceptible of improvement by treatment but at the same time in some cases leaving behind sufficient brain damage to nullify any real clinical effect. Patients with low cell counts (an inactive fluid), on the other hand, have a relatively static process, less susceptible of improvement by treatment, but at the same time less likely to result in clinical progression. The correlation is definite enough to be of some prognostic value but not sufficiently great to permit withholding adequate treatment from any patient with general paralysis who has not previously had treatment known to have been adequate.

Pre-treatment spinal fluid protein levels tended to show the same general relationship to clinical outcome as did cell counts, but the correlation was less definite. 
In Fig. 8 is shown post-treatment cell count at 1, 3, and 5 years in relation to pre-treatment cell count. These findings are essentially similar to those previously reported in asymptomatic neurosyphilis treated with penicillin. Elevated cell counts of 11 or more had almost entirely disappeared by the end of

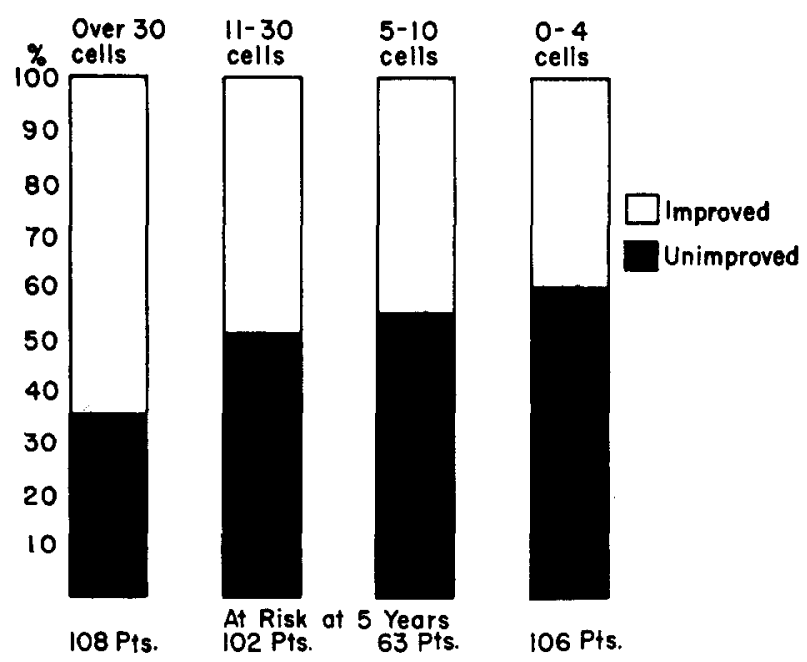

Fig. 7. - Per cent improved 5 years after treatment in relation to pretreatment cell count.
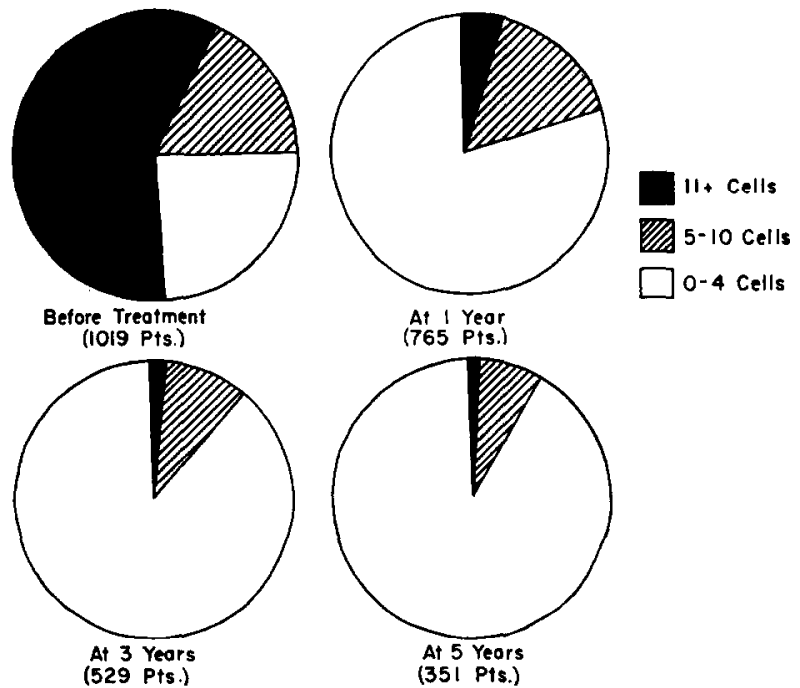

Fig. 8.-Post-treatment cell count in relation to pretreatment cell count.

the first post-treatment year. In contrast as shown in Fig. 9, elevated protein levels of $51 \mathrm{mg}$. per cent or more persisted in 10 per cent of patients at 5 years after treatment. 
Since persistently abnormal cell counts of 11 or more cells were not encountered with any frequency after the treatment of paresis with penicillin, they could not be correlated with clinical outcome. Borderline cell counts of 5 to 10 were observed in a significant number of patients 3 years after treatment, at which time clinical progression was recorded in 18 per cent of this group as compared with only 6 per cent of the group with 0 to 4 cells. Although abnormal and borderline protein levels, as shown, persisted with much greater frequency than did elevated or borderline cell counts, post-treatment protein levels could not be correlated with clinical outcome.
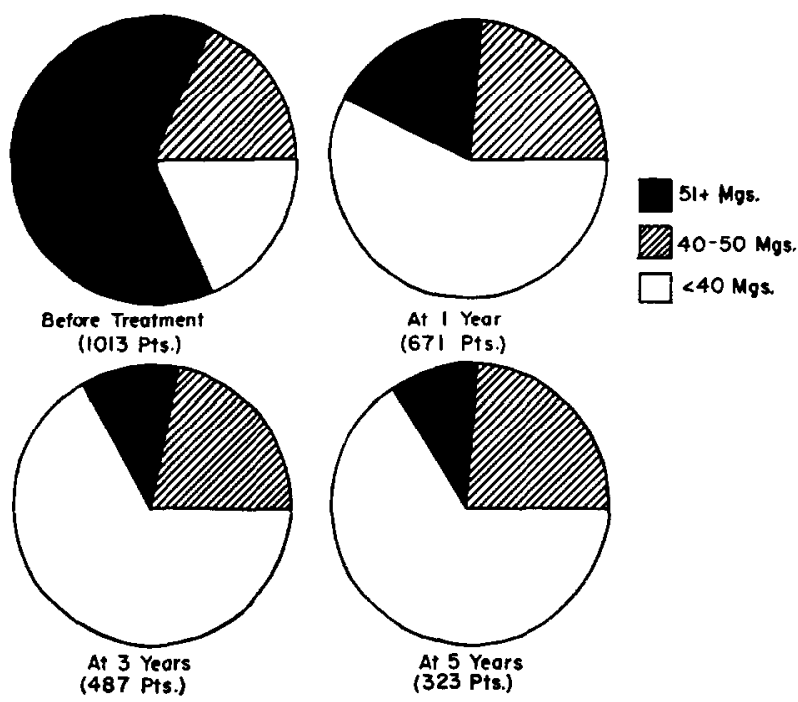

Fig. 9.-Post-treatment protein in relation to pretreatment protein.

Let us turn now to a consideration of outcome in relation to treatment received. Outcome was significantly affected by treatment given prior to entry in to the study. The effect of previous penicillin therapy could not be evaluated because only 56 patients were known to have received this drug previously. At 6 years after study treatment there was a significant difference in outcome between the 327 previously untreated patients and the 483 patients previously treated with therapy other than penicillin. Improvement occurred in only 42 per cent of previously untreated patients, as contrasted with 58 per cent of previously treated patients. Differences of like degree were found with respect to ability to work and psychiatric institutionalization at 5 years after treatment.

In view of the large percentage of previously treated patients and of the demonstrably favorable effect of previous treatment, the present material cannot be said to reflect the effect of penicillin alone, but only the effect of penicillin with or without fever therapy on a mixed group of cases with general paralysis, a significant but undetermined number of whom already had an at least partially arrested process when penicillin was first given. That this may detract from the 
accuracy of the absolute percentages quoted cannot be gainsaid, but that it significantly affects their order of magnitude seems unlikely.

The present material was not found to be suitable for a biostatistically valid comparison of the effects of aqueous and repository penicillin. Relatively few patients received the long-acting preparations, and these were largely an ambulatory group. Among severe cases the results with repository penicillin seemed somewhat better than with aqueous penicillin, undoubtedly due to the difference in the type of patients treated rather than the type of penicillin used. It would appear, however, that repository penicillin is at least as efficacious as aqueous penicillin for the treatment of general paralysis. This conclusion has been amply borne out by our clinical experience with patients treated more recently than those included in the present study.

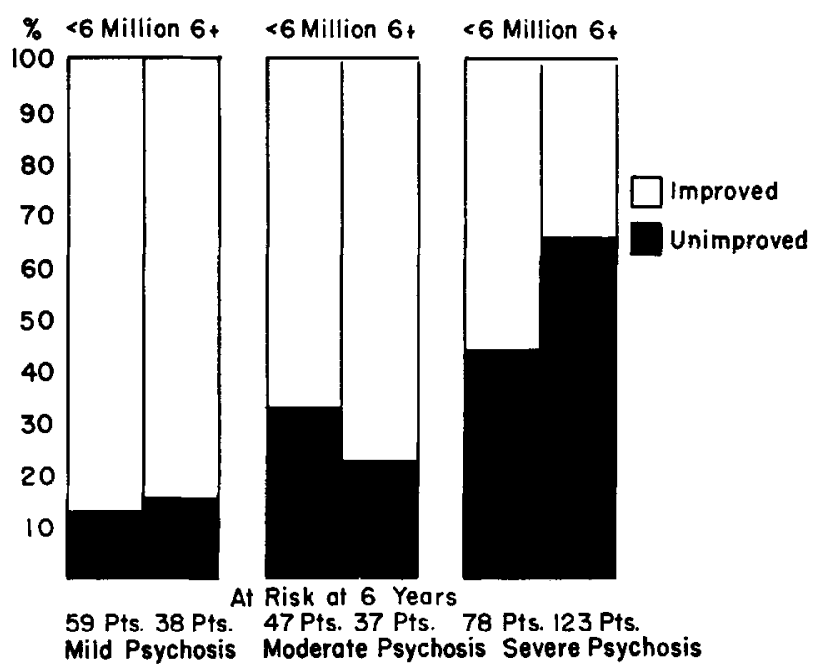

Fig. 10-Clinical status 6 years after treatment in relation to amount of penicillin.

In Fig. 10 is shown clinical status 6 years after treatment in relation to amount of penicillin given as study treatment. Consistent differences in clinical outcome could not be demonstrated. Likewise, consideration of work status and psychiatric institutionalization in these two treatment groups failed to reveal consistent differences. These data fit in well with the known extreme sensitivity of Treponema pallidum to penicillin. Although the present material does not permit a statement as to the minimal effective dosage of penicillin in general paralysis, it does permit of the pragmatically important conclusion that 6 million units is probably ample.

A comparison of the effect of penicillin alone with the effect of penicillin plus fever therapy is of utmost practical importance. Unless it can definitely be shown that there is meaningful additive effect, the markedly increased hazards and expense of fever therapy preclude its routine utilization. 
In Fig. 11 is shown the clinical status 5 years after treatment in patients treated with penicillin alone and in patients treated with penicillin plus fever therapy. At 5 years after treatment the percentage improved was practically identical for the two treatment groups among the severe, moderate, and mild cases.

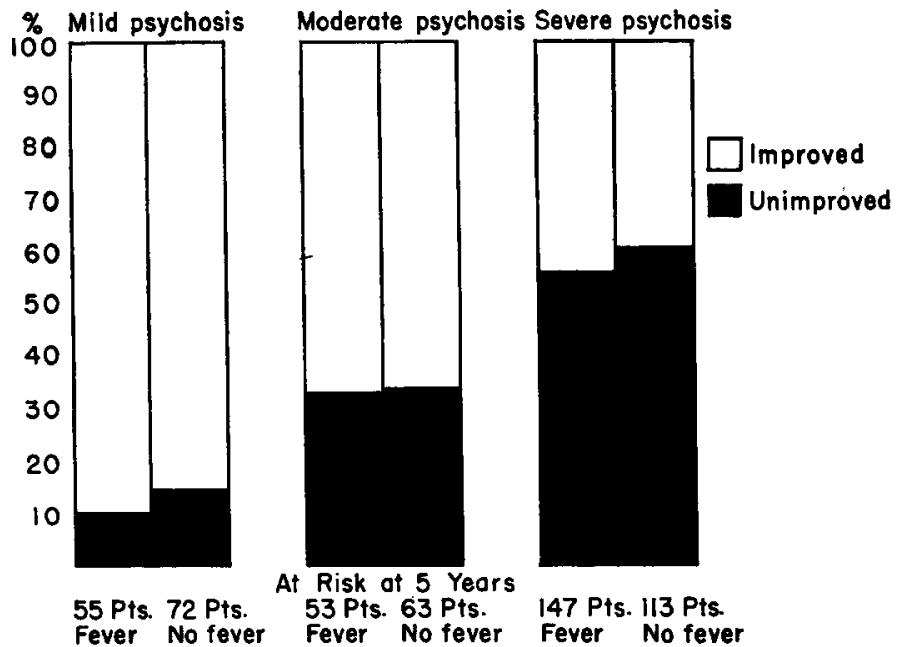

Fig. 11.--Clinical status 5 years after treatment. Penicillin versus penicillin plus fever.

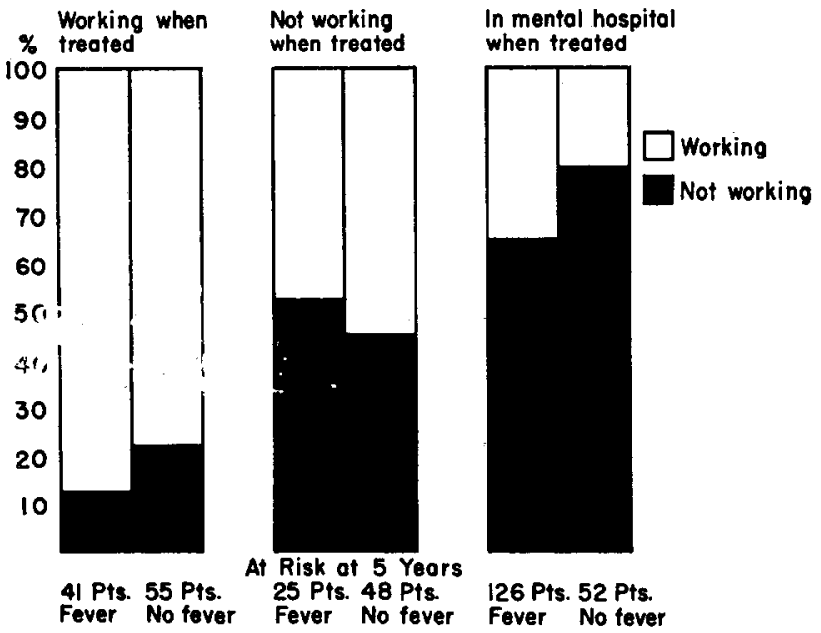

Fig. 12.-Per cent able to work 5 years after treatment. Penicillin versus penicillin plus fever.

In Fig. 12 there is shown the per cent of patients able to work 5 years after treatment among those treated with penicillin alone as compared with those treated with penicillin plus fever. Arnong the two groups of patients not in a 
psychiatric institution at the time of treatment, consistent and significant differences in work status 5 years later were not observed. However, patients in a psychiatric institution when treated, who constituted numerically by far the largest group treated with fever, did better than similar patients treated with penicillin alone, 35 per cent as compared with 20 per cent having returned to work 5 years after treatment.

In general, as measured by the two yardsticks of clinical mental status and work or institutionalization status, it seems clear that the possible additive effects of fever therapy are not sufficient to justify its use in any significant number of patients. Yet, the individual clinician has some grounds for its use in selected cases - the avoidance of clinical Herxheimer reactions from penicillin when the use of this drug is delayed until the patient has had a paroxysm or two of fever; and the greater rehabilitation for work among fever plus penicillintreated institutionalized patients.

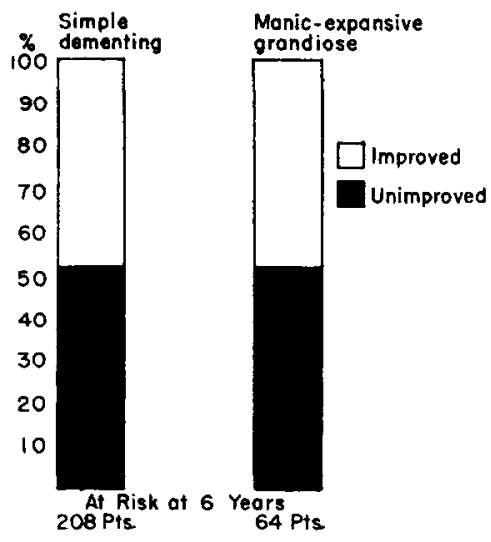

F1g. 13. - Per cent improved 6 years after treatment in relation to type of psychosis.

In Fig. 13 is shown the per cent of patients improved 6 years after treatment in relation to type of psychosis. As shown, outcome in these two groups was essentially identical with respect to per cent improved, but rehabilitation for work was achieved in almost half of the simple dementing group as compared with a little better than a quarter of the manic expansive grandiose group. Depressed patients did relatively well and paranoid patients least well, although in these groups the number followed was too small to allow of quotation of actual percentages. As expected, the small groups of patients diagnosed as paretic on somatic grounds alone and those with only questionable psychosis did well according to all criteria of outcome. It seems clear that type of psychosis is of less importance prognostically than is severity of psychosis, and that the patients with so little mental aberration as to fall in to the "questionable" or "somatic only" categories have the best prognosis. The importance of early diagnosis and prompt treatment of general paralysis is thus once again demonstrated. 
In Fig. 14 is shown the per cent of patients improved 5 years after treatment in relation to duration of paretic symptoms at time of treatment. As would be expected, patients with symptoms of less than one year's duration seemed to do slightly better than did patients with longer duration at time of treatment. Further subdivision of the former group yielded no consistent differences. It is felt that the actual differences are probably even more striking than the demonstrated ones and that the unreliability of historical data as obtained from paretics and/or their families may somewhat obscure these differences.

In Fig. 15 is shown the per cent of patients able to work 5 years after treatment in relation to duration of symptoms at time of treatment. The same general relationships are shown, with the ability to work inversely proportional to

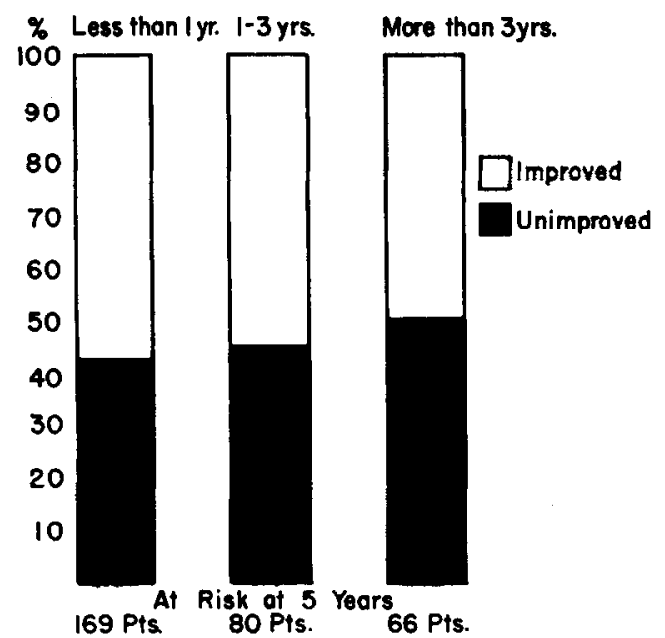

Fig. 14. - Per cent improved 5 years after treatment in relation to duration of paretic symptoms at time of treatment.

the duration of symptoms at the time of treatment. These data once again support the conclusion that early diagnosis and treatment afford a significantly increased prospect of clinical improvement, rehabilitation for work, and maintenance in or return to community life.

Outcome was considered in relationship to a number of other factors including age, race, and sex, educational level, chronic alcoholism, and the presence or absence of a Herxheimer reaction, Age, race, and sex had little influence on outcome, and chronic alcoholism and educational level almost none. Of particular importance is the question repeatedly raised in the past of possible permanent damage from the Herxheimer reaction. In the present material there is no evidence of such permanent damage other than the single death in status epilepticus. Indeed, patients who experienced either clinical or febrile Herxheimer reactions had a more favorable outcome as measured by clinical improvement or work status than did patients who did not experience the Herxheimer reaction. These differences may be in part due to the relatively small size of the Herxheimer 
groups, but in part are due to the fact that Herxheimer reactions occur with greater frequency in patients with an active inflammatory process of the central nervous system, which has been shown to be more susceptible of improvement by treatment.

The present material, despite its size, was found not to be suitable for a detailed comparison of the value of repeated courses versus one course of penicillin. Many patients were given repeated courses as part of the original treatment plan without regard to any real indications for re-treatment. Others were re-treated for "insufficient improvement" even though the paretic process was apparently static, and still others received further treatment for other reasons. In all, 289 of the 1,086 patients were given more than one course of penicillin for one or another reason, but only 50 patients were re-treated for apparent clinical progression, of whom 45 had sufficient follow-up to provide a basis for analysis.

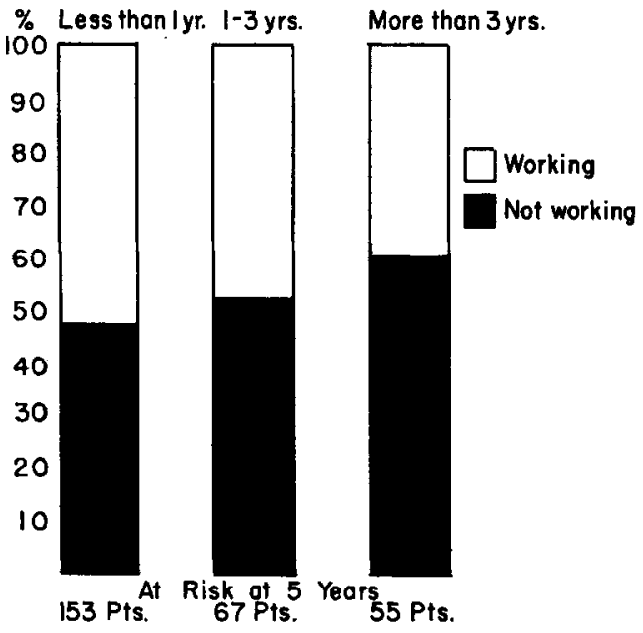

Fig. 15.-Per cent able to work 5 years after treatment in relation to duration of paretic symptoms at time of treatment.

Re-treatment seemed to benefit 14 patients who had improved after the initial course and subsequently had progressed, as only two of them were dead or institutionalized at latest observation. Re-treatment did not seem to benefit 31 patients who had not improved after the initial course, as 26 of them were dead or institutionalized at the latest observation. It is of interest that all of the 14 patients who had improved after the initial course of treatment did so on a dose of less than 6 million units of penicillin, only subsequently to progress. The paretic process in this group was obviously an active one, temporarily favorably affected by a subcurative dose and therefore susceptible of being arrested by re-treatment. In contrast, 16 of the 31 patients who had not improved after the initial course of treatment had received six or more million units of penicillin, suggesting that in this group the process was advanced and static and insusceptible of improvement by any amount of additional penicillin. 
Since the presence or absence of the more frequent signs and symptoms of paresis was recorded both at the time of study treatment and at each post-treatment observation, it has been possible to evaluate the effects of treatment on these signs and symptoms individually, a matter of considerable importance to the physician dealing with these patients.

In Table $\mathrm{V}$ is shown the effects of treatment on individual signs and symptoms at the time of treatment. Significant numbers of patients were followed for 5 years. It should be clearly stated that in order to avoid, as far as possible, subjective bias and differences between examiners, no attempt was made to record improvement, but only complete disappearance of the sign or symptom in question. In view of this rather stringent interpretation, the effects of treatment upon the symptoms and signs of paresis as shown in Table $\mathrm{V}$ seem to be in general strikingly beneficent. Of considerable interest is the relative resistence of impairment of judgment, calculation, information, insight, and speech as compared with the other symptoms of paresis.

Table V. Effect of Treatment on Individual Signs and Symptoms Present at Time of Treatment

\begin{tabular}{ll}
\hline & $50 \%-60 \%$ Cleared up at 5 Years \\
Disorientation & Depression or euphoria \\
Convulsions & Untidy dress \\
Tremors & Incontinence \\
Inability to perform personal toilet & \\
& $40 \%-50 \%$ Cleared up at 5 Years \\
Impaired memory & Inpaired retention \\
Impaired handwriting & Delusions, hallucinations \\
Illogical conversation & Psychotic on simple obs. \\
& $30 \%-35 \%$ Cleared up at 5 Years \\
Impaired judgment & Impaired calculation \\
Impaired insight & Impaired information \\
Impaired speech &
\end{tabular}

\section{SUMMARY}

Paresis strikes most frequently during life's most productive years. It is seen approximately three times as frequently in males as females, and over twice as frequently in whites as in Negroes. The usual incubation period is 10 to 24 years after infection. The simple dementing type of psychosis is more frequent than all other types combined. In the absence of previous treatment the spinal fluid complement fixation reaction is always positive and the spinal fluid cell count is almost always elevated. The blood serologic test may, rarely, be negative.

Penicillin prevents paresis. None of the present group of 1,086 patients had received penicillin for early syphilis. Paresis has not been observed to develop following the penicillin treatment of asymptomatic neurosyphilis. 
The present study cannot be said to reflect the effect of penicillin alone, but only the effect of penicillin with or without fever therapy on a mixed group of paretics, a small but undetermined number of whom already had a process perhaps wholly or partly arrested by other types of therapy before penicillin was given.

The trend in recent years in the United States has been toward the treatment of paresis with penicillin alone. The possible additive effects of fever therapy are not sufficient to justify its use in any significant number of patients. The early diagnosis and prompt penicillin treatment of incipient paresis result in clinical remission and ability to work in more than 80 per cent of patients and will prevent practically all deaths from neurosyphilis. Even the severe or institutionalized patient has one chance out of three of improvement and rehabilitation for work. In contrast to the dire prognosis of untreated paresis only 9 per cent of penicillin-treated paretics are dead of paresis 10 years after treatment. Even so, the penicillin-treated paretic has nearly four times the death rate of his or her nonsyphilitic age, race, or sex counterpart.

The effects of treatment upon the individual signs and symptoms of paresis are in general strikingly beneficent. In the majority of cases, however, abnormalities of speech, insight, calculation, judgment, and general information do not entirely disappear.

Repository penicillin is quite as suitable as aqueous penicillin. Although the minimal effective dose is unknown, the present study suggests that 6 million units is probably ample.

In general, more than one course of penicillin is of no demonstrable additional value. Absence of clinical improvement, persistently abnormal spinal fluid protein level, or persistently positive spinal fluid complement fixation reaction are not indications for re-treatment. Re-treatment with penicillin is indicated under the following circumstances: an initial course of therapy of less than 6 million units, temporary clinical improvement following an initial course with subsequent clinical progression, or spinal fluid cell count of 5 or more after the first post-treatment year. The essentially identical probability of death in patients who receive one course and patients who receive more than one course of treatment is strong evidence against the value of routinely repeated courses of penicillin in the treatment of general paralysis.

Herxheimer reactions, notably exacerbation of the paretic psychosis or convulsive seizures, occur more frequently in the presence of spinal fluid pleocytosis than in its absence. The Herxheimer reaction does not appear to cause long-term damage.

Skilled neuropsychiatric examination affords definite prognostic data as to the probability of improvement in psychosis, rehabilitation for work, and to a certain extent as to death itself. Progression and death due to neurosyphilis occurred almost altogether in patients with "severe" pretreatment psychosis. Post-treatment psychiatric hospitalization was directly proportional, and clinical improvement and rehabilitation for work inversely proportional to the severity of pretreatment psychosis. Type of psychosis was of less importance prognostically. The shorter the duration of psychosis at the time of treatment the more 
frequent was clinical improvement and rehabilitation for work. The presence at the time of treatment of incontinence, inability to perform simple acts of personal toilet, and of convulsions afforded a particularly poor prognosis for longevity.

Work status at the time of treatment was of great prognostic import, and of perhaps equal significance was the length of time which had elapsed since the patient had been able to work at his usual occupation.

Age, race, and sex were relatively unimportant factors in prognosis.

Pretreatment pleocytosis indicates an active inflammatory process more susceptible of improvement by treatment, but in a minority of patients leaving behind sufficient brain damage to nullify any real clinical effect. The absence of pleocytosis indicated a relatively static process less susceptible of improvement. Pretreatment spinal fluid protein levels were of less prognostic import. Persistent cell counts of 11 or more were for practical purposes not encountered after penicillin treatment. Clinical progression did, however, occur significantly more frequently with persistent cell counts of 5 to 10 than with cell counts of 4 or less. Persistent spinal fluid protein elevation was relatively frequent but of no prognostic import.

Considering the fact that as the incidence of syphilis itself decreases, dementia paralytica is becoming something of a neuropsychiatric rarity, the data which I have presented are perhaps of more historical interest than practical importance. They are, however, a dramatic illustration of the progress of medicine in four decades. A disease which was uniformly fatal within a few short years prior to Wagner von Jauregg's 1917 discovery of the efficacy of malaria therapy has now become curable in some, and partially remediable in many, by virtue of brief treatment with penicillin. The credit for this accomplishment is shared by your country through Fleming and Florey, and my own through Mahoney. Most of it belongs to Britain. May our future scientific and friendly brotherhood prove as profitable! 